\title{
A Report on Designing of Wireless Sensor Networks for IoT Applications
}

\author{
P. Ramchandar Rao, S. Srinivas, E. Ramesh
}

\begin{abstract}
Internet of things is defined in many ways is an internet technology connected to devices, machines and tools by the means of wireless technology, such as connected homes, cities, cars and roads, etc. The IoT is the platform to connect physical objects that contain built-in technology to interact and sends or communicate with their internal blocks or external environment. Nearly one trillion devices are internet connected devices which are available with mobile applications, linking all these connected things. Billions of devices are being communicated all the way though out the world over network protocols, in 2010 which was made by an IoT. Daily around 12.5 billion things or devices are interacting with internet. Since past two decades many researchers and industries were attracted towards IoT because of its reliability. If domestic appliances like Oven, Refrigerator, Air conditioner, Geezer, Smart TV are connected in a network, they all work together in association to provide an ideal service as an entire, not as a collection of independently working devices. IoT is a powerful tool which is used in many ways in the development of real-world applications and services, for example building a smart residence where light should $\mathrm{ON}$ when the person enters into the room at night time, windows can be closed automatically when it rains, and automatically windows will be open if any leakage of gas. Wireless Sensor Networks are included into the "Internet of Things", where sensor nodes are connected to the Internet vigorously, and use it to act as a team and complete their action. Wireless Sensor Networks are well apt for long-term environmental data acquirement for IoT representation. In this paper, we provide the information on designing challenges on wireless sensor networks for an IoT application.. This paper presents an overview on an IoT, functional design of WSN for IoT application, details an architecture of IoT and related key issues.
\end{abstract}

Index Terms - Energy efficiency, IoT, IoT Application, Sensor Node, Privacy, Security, Wireless Sensor Network.

\section{INTRODUCTION}

Interconnection of built in computing devices within the accessible Internet structure is defined as Internet of Things. Mostly, IoT is an advanced connectivity of devices, systems, and services that goes ahead to machine-to- machine (M2M) communication which covers a range of protocols, domains, and applications. The interconnectivity of all these embedded devices includes smart objects, such interconnection among the devices is expecting to rule automation in all fields enabling complex applications. Using this technology we can enable objects to communicate with each other to perform the desired action even in the absence of human interference. IoT consists of sensor, actuator, controller, Internet, and connecting devices. Despite these important benefits, a strong argument is heard like the more interconnectivity between the devices in Internet the high it can create security risks and privacy risks. The following issues were highlighted when a survey was conducted on IoT, they are (1) Misuse of personal information through unauthorized access, (2) incorporate threat on other systems and (3) enabling security risks. Even though such security and privacy risks are existing in the conventional computers and networking too but incase of IoT it is even more heightened when compared to conventional computers.

Generally, when Iot devices are connected to Internet, the chances of accessing and misusing the personal information by the intruders will be more which may result in security issues. Security risks will be more in smart devices which may store sensitive information like bank account information, passwords and other types of information, Intruders could take advantage of vulnerabilities to facilitate identity theft or fraud. Thus, more we install the smart devices in our home more the consequences we need to face where an intruder can easily access the personal information to misuse.

The intial concept behind Iot was to have an interchange of information between the devices fueled by the Radio Frequency Identification, Nano-technology and WSN. Sensors measures surrounding information and converts to electrical signal which is further send to controller, an automated action is performed based on the decision taken by the controller.

The below given figure gives the annual report on M2M connections will account for more than half of the world's 28.5 billion connected devices by 2022 .
Revised Manuscript Received on July 12, 2019

P. RamchandarRao, Assistant Professor, Dept. of ECES R Engineering College, S R Engineering College, Telangana, India (e-mail: Ramchandar_rao_p@srecwarangal.ac.in)

S. Srinivas, Assistant Professor, Dept. of ECE, S R Engineering College, Telangana, India (e-mail:srinivas_s@srecwarangal.ac.in)

E. Ramesh, Assistant Professor,Dept. of ECE, S R Engineering College, Telangana, India (e-mail: e_ramesh@ srecwarangal.ac.in) 


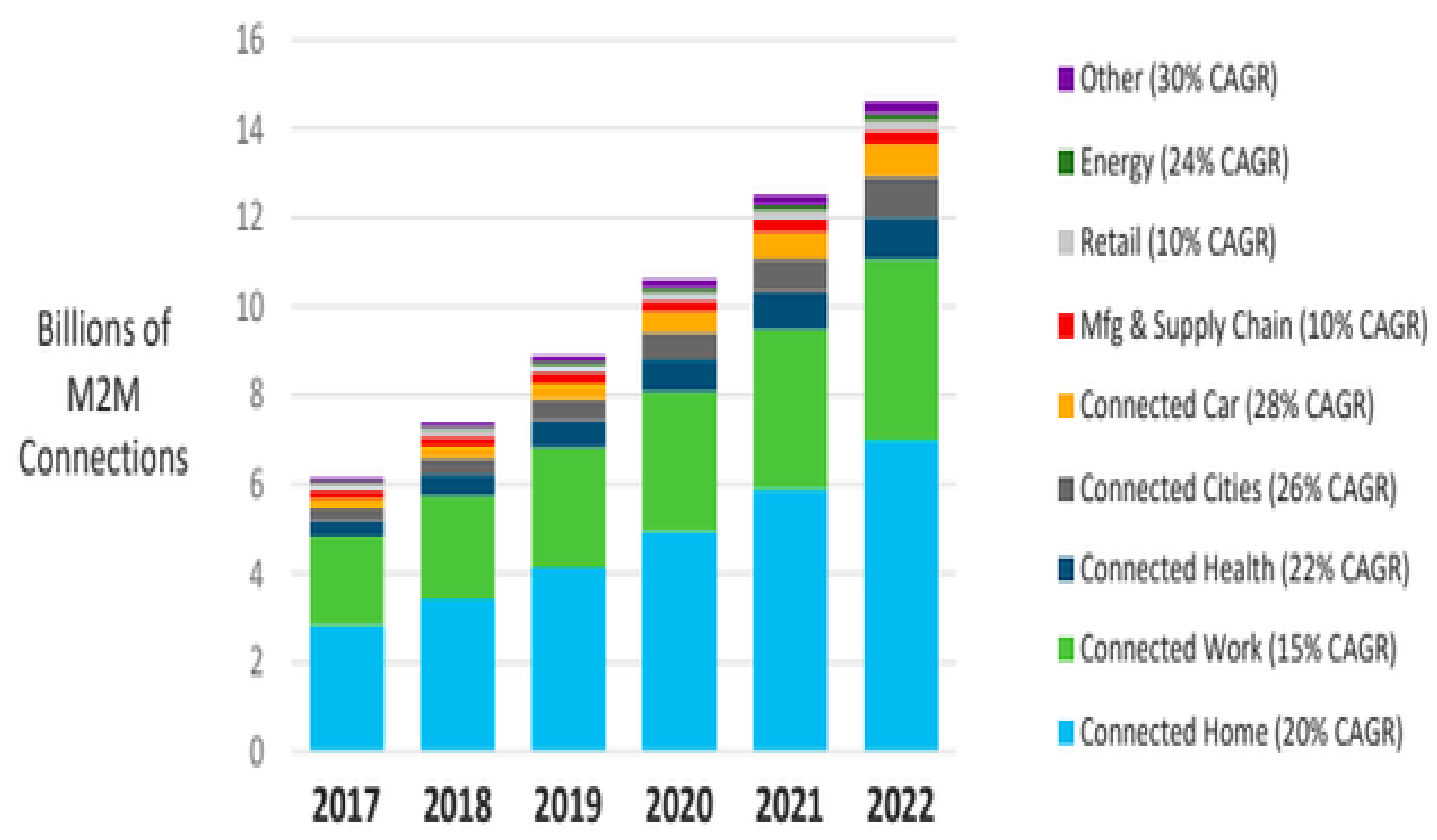

Fig.1 Global M2M connections/IoT Growth by 2022, connected home largest, according to survey of Cisco

\section{SURVEY ON IOT}

Definition: There is no specific definition towards IoT, the number of definitions so given on IoT is based on their individual experiences on IoT. The Internet of Things is generally meant for an open and comprehensive network of intelligent objects (smart devices) that have the capability to auto-arrange (controller), exchange of data, data and resources,(cloud) reacting and acting(actuators) to handle the situations and changes in the environment. IoT is growing and continuing in the world of IT to an extent. Over the past decades IoT has gathered IT attention by projecting its vision of a global infra and networked physical objects which are interconnected and then enabling them to access at anytime anywhere. The IoT is not only meant for objectobject connection instead it can have a connection between human-human and human-thing throughout the global network which provides each and every object or human a unique Id. IoT is a smart and intelligent technology which connects to and communicates with anything in an intelligent way which has been seen ever before. Generally connectivity refers to the connections among the desktops, servers, laps, tablets, mobiles and smart phones. But, in case of IoT connectivity refers to the connection of sensors, actuators which are embedded in physical objects through some wired or wireless networking using same Internet IP. These networks churn out huge volumes of data for analysis.

Vision of Internet of Things:

Vision of IoT is considered in two ways. 1) Internet point of view and thing point of view. From the internet point IoT involves the services of Internet through which desired actions can be performed by the things and its surroundings. Thing-centric involves the connectivity of different things which are accessed by the Internet.

Challenges of IoT:

Energy Efficiency
Security

Scalability

Bandwidth Management

Modeling and Analysis

Interfacing

Interoperability

Data storage

Data Analytics

Complexity management

\section{ARCHITECTURE}

Conventional Iot architecture is proposed with three layers namely Perception, Network, and Application layers. Later one more layer is added to the list, is called Middleware layer in between application and network layers.

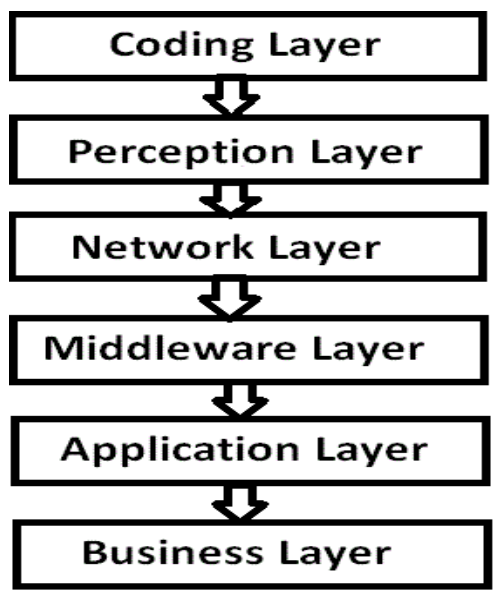

Fig.2 Architecture of IoT 
In proposed architecture some more layers are included using the finest features of IoT model, Internet and TMN are based on TCP/IP models respectively.

A. Coding Layer: The first layer of IoT architecture is Coding layer. In this layer reach and every object is assigned with a unique Identification number.

B. Perception Layer: Perception layer is the device layer in this several digital and analog sensors like RFID, IR, Temperature, Humidity, Light, speed and location etc are used to sense the useful information from physical environment and converts into electrical or digital signal first and then again converts to digital signals using ADC's later which is given to network layer for further processing.

C. Network Layer: All the smart and network devices, servers are connected to internet in the network layer. Its characteristics are also used for transmitting and processing sensor data through Wi-Fi, Bluetooth, WiMaX, Zigbee, GSM, 3G, 4Getc with protocols like IPv4, IPv6, MQTT,
DDS etc. In what way the devices are connected to a network is given in this layer.

D. Middleware Layer: It is also called as processing layer. Middleware layer processes the data received from the network layer and stores the data into database which includes the technologies like Cloud computing, servers. The controller analyzes the data and takes decisions according to the received information and sends the information to application layer. It also manages and provides services to the lower layers.

E. Application Layer: This layer comprises of custom applications which provides specific services to the users. Smart homes, transportation, garden etc., could be the IoT applications,

F. Business Layer: The business layer manages the whole IoT system, including applications, business and profit models, and users' privacy. Different business models are generated in this level.

\section{An Example loT Implementation}

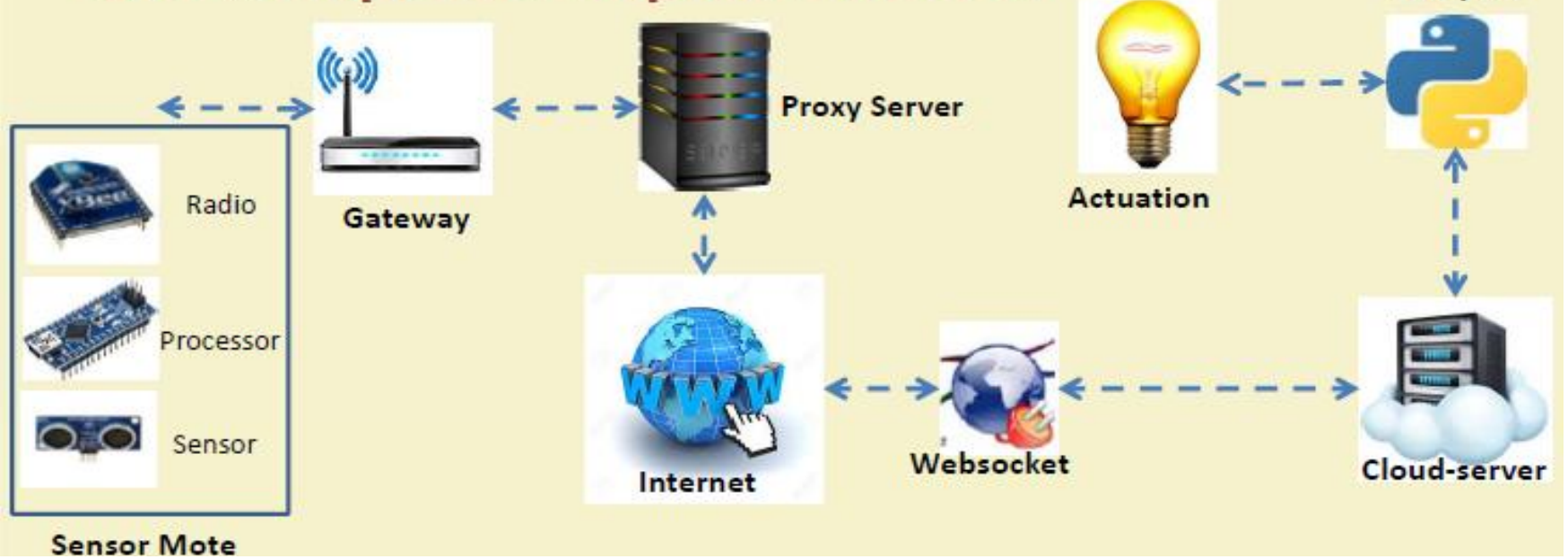

Fig. 3 Example for IoT Architecture with different layers

\section{DESIGNING CHALLENGES}

When we design an IoT application we need to focus on many issues like energy efficiency, stability of network, network failures, memory management, operating system optimization and tuning, cost effective, handle flaws and all sensors will trigger the action in time or not etc.

\section{Energy Efficiency:}

In energy efficiency point of you overall energy consumption by sensors and wireless networks must be minimum when power fluctuations. To increase the energy efficiency in IoT selection of sensors and components and software design.

For example we have a set of tasks and we have assigned some sensors for each and every task. So which sensor should work at what time and if a time interval is given to a sensor and once if the work is done it should go to inactive state, which improves the efficiency of the system.

\section{Stability of Network:}

As our system works on the basis of interconnection of things and their communication with the help of internet, we need to specify the protocols specifically as to overcome the signal problems. The network signal should be very strong.
In order to overcome such issues we need to deploy IoT products need strong network, write enhanced algorithms, and dynamic detections.

\section{Network Failure and Memory Management:}

As we are working internet ant its connecting devices, unexpectedly if any network failure occurs the IoT product may not work efficiently. In order to overcome this we need to store critical data in internal non-volatile memory. We need to choose right memory and data structures. In [15] the authors introduced power network monitoring using embedded Web server. The system employs LPC2148 and embedded TCP/IP Rabbit Core Module 5170.

\section{Security and Privacy:}

Internet is possibly the victim of its success as far as security is concerned. Security measures the absence of threats which in turn measures the absence of fear. Privacy measures the absence of misusing the personal information by the intruders.

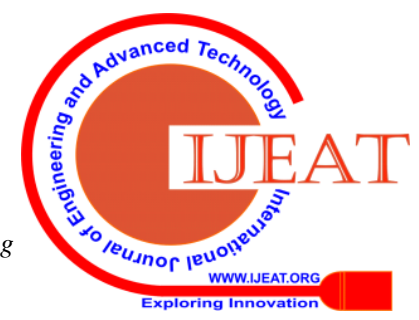


If an human is wearing any IoT device it should not be tracked by the intruder as to preserve privacy.

\section{Wireless Sensor Networks:}

At many times, it is not possible to work with a single sensor, we may need many number of sensors and also the interaction between the sensors is required. But, the technologies what we are using like Bluetooth, Zigbee, RFID, NFC are non-IP technologies and it covers a small range. To monitor a large area more number of sensors is required. Wireless sensor networks consist more number of sensors connected with the help of wireless technologies. The data collected by the sensor nodes is send to the network protocols which further relay the information on the cloud over the internet. Star, Mesh and Hybrid are the most popular network topologies used in Wireless sensor networks. The standard used in Wireless sensor network communication is IEEE 802.15.4.

The IoT applications requirements are long life low cost and reliable, unless reusable hardware and software platforms are available, including flexible Internet enabled servers to collect and process the field data for IoT applications.

\section{Applications of Wireless Sensor Networks:}

In wireless sensor network applications the bi-direction device is connected to sensors in a multi-hop manner. In any application several sensor nodes connected, these are measure the physical environment data like temperature, light, humidity, speed and etc., then pass the information to processing device. A typical sensing node is shown in the figure below.

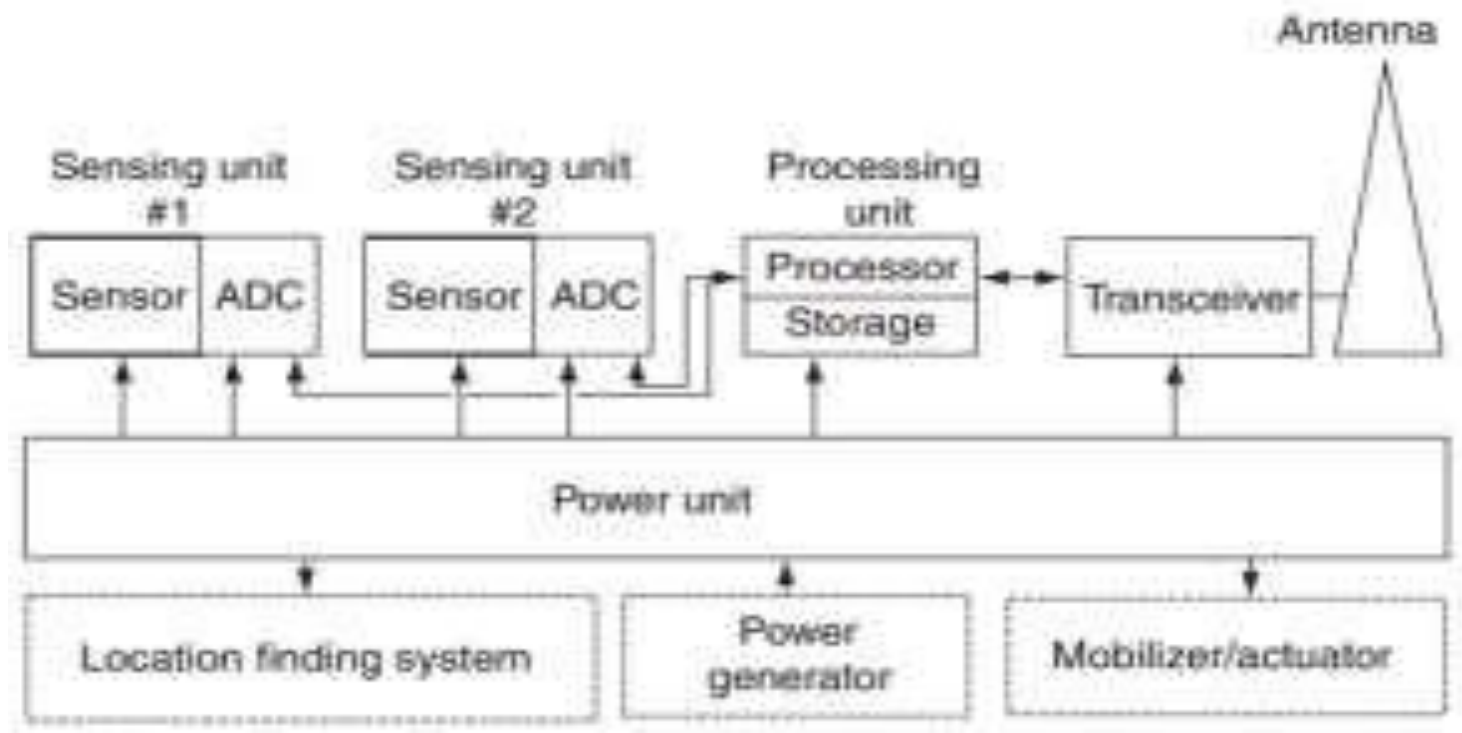

Fig.4. Typical sensing node

The sensing nodes communicate in multi-hop channel, each sensor having transceiver antenna, a processing device microcontroller and an interfacing circuit to actuators along with power supply which either battery or any other energy harvesting technology. To save the processing data an addition unit i.e., memory unit storage is added with a part of the sensing node.

Some of the applications of wireless sensor networks are military applications, health monitoring, environmental like earth sensing's, air pollution monitoring, forest fire detection, landslide detection, water quality monitoring and industrial monitoring.

To resolve the problems number of possibilities that solutions are proposed by wireless sensor network technology and RFID technology when integrated together.

Intel Research Labs provides an example solution for Wireless Identification Sensing Platform (WISP). WISP is an inactive wireless sensor network which has been built with some sensors like light, temperature and light gauges. Although the functioning of both WSN and RFID the one and same but differs in the range such that RFID has a minimum range whereas WSN sensor network has a long range. More number of sensors is connected in WSN network whereas only some sensors are connected in RFID sensor network. Peer-Peer communication is done in WSN
Sensor Network whereas a non symmetric communication is done in RFID Sensor Network. Moreover most of the WSNs are based on the IEEE 802.15.4 standard, which specifies the physical and MAC layer of Low-Rate Wireless Personal Area Networks (LR-WPANs).

Many solutions have been proposed including 6LOWPAN standard that enables IPv6 packets to be transmitted through the network which are computationally restricted. Technologies that integrate IoT with WSN are a very hot research topic.

Main intention is to optimize energy consumption to progress its competence. Large amount of information is stored the database which is been collected and stored in it by the sensors. Efficient data mining algorithms needs to be implemented to fulfill user expectations as the user may require short and useful information. Extracting limited and useful data from the huge amount of database is called data mining technology. As we are using sensors, energy consumption will be more if sensors are active all the time. In order to optimize energy consumption switches the sensors from dynamic to inactive and from inactive to dynamic based on the user requests. As the collected

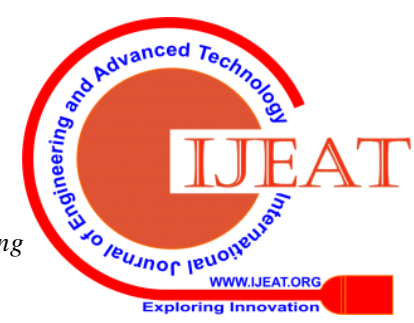


information is stored in the Cloud, the Cloud is to be accessed only by the authorized user. Strong authentication techniques are used to access the cloud such that no other unauthorized user may not access the cloud.

\section{APPLICATIONS}

All the household application what we are using now a days are smart appliances like smart TV, smart phone, smart washing machine etc. but all these appliances will work only when human interacts with them physically. But IoT is the one which will make all the appliances to work themselves without the interaction of human with them. No human intervention is required. IoT applications are some innovative applications will work with some sensors, actuators and controllers etc.

Smart Home: Using IoT we can control all the household appliances remotely. For example mostly when we turn on a light we may forgot to turn it off, or when switch on the TV we may again for get to switch off which may result to energy wastage. And also when we on the motor to fill the water tank we may forget to switch off which may result in wastage of water. To overcome such scenarios IoT based applications are used. With the help of LDR and PIR, sensors will work and automatically will detect the human presence or absence and put either the light will on or off.

Smart Gardening: Generally gardening requires water, soil moisture levels and continuous observation to see the levels of moisture, water levels etc. Smart gardening checks the moisture level(dry/wet), according to the moisture level the motor will function(on/off).in some cases temperature and humidity sensors aloso require to monitor the garden.

Smart Environment:Natural disasters like floods, droughts, earthquakes, tsunami etc can be detected with the help of IoT based applications which may be used to give precautions. IoT monitoring system is used to measure the pollution in air which in turn predicts the depletion of ozone layer. [17] In smart cities garbage monitoring system is used to monitor the day to day garbage in trash bins and alerts the municipal corporation if the trash bin is full with the help of GSM modems.

\section{Smart Hospitals:}

All the corporate hospitals will be prepared with smart wearable tags which are inbuilt with RFID which will be given to patients. Tag monitors the patient's condition like pulse rate, BP, fever, etc. Each and every patient is allotted with one RFID, from this RFID the doctor can monitor the condition of the patient on his desk. In [16] authors presented health should be given more importance in person's life. Health monitoring systems has been developed in these few years that can increase in providing better health.

\section{Smart Transportation:}

As how the population in our country is increasing day to day the problem of transportation is also increasing in the same way. In the present transportation scenario transportation is leading its role in the cities. Both the private and public transportation has become the key issues for the government.
To overcome transportation problem metro, local trains are giving their best in the cities. So IoT has brought the concept of smart transportation which may increase the transport facility for better life. Some of the examples like periodic traffic forecast to plan a trip/journey, Dynamic car pooling / car sharing where number of passengers on the same roué can travel through only one transport to optimize traffic the and also passengers can pay the amount through app in the smart phone instead of money exchange. GPSbased car tracking where we can track the vehicle and optimize the ridership, dedicated short-range communication (DSRC) enabled vehicular communication system, route optimization to reach the destination early along with the traffic information, Metros are even equipped with sensors and actuators for the reliable, comfortable and safe journey. Easy navigation and safety measure can be achieved with the help of smart transportation since the drivers as well as passengers can track/navigate the route.

Smart lighting system: Depending upon light intensity, street lights at night hours it should be $\mathrm{ON}$ and day time it should be OFF, it saves the power, if we use solar panels, to store energy during night hours light utilizes the stored power.

Some more modern applications of IoT are smart parking, smart phone detection, smart lightening system, waste management, liquid pressure, water management and control system, radiation levels, intelligence shopping applications, smart product management and ete.,

\section{CONCLUSION}

Finally, this paper concludes that IoT applications are used in number of domains like household applications, productions, industrial, transportation, education, governance, mining, habitat etc. Even though IoT has abundant benefits, there are some flaws in the IoT like insecure web interface, insufficient authentication/authorisation, insecure network services, lack of transport encryption and privacy concerns. The key comments in the literature are that, there is no concerned definition is given for IoT, Some standards are to be maintained at architectural level, as per the needs of the user IoT applications needs to be updated. The proposed system implements energy efficiency, network failure, memory management, security and privacy issues for good performance of system.

\section{REFERENCES}

1. J. A. Stankovic and et al., "Research directions for the Internet of Things," IEEE Internet Things J., vol. 1, no. 1, pp.3-9, Feb. 2014.

2. Zhengguo Sheng, and et al., "Recent Advances in Industrial WSN's towards Efficient Management in IOT”, vol. 3, 2015.

3. HuanshengNing, and et al., "Aggregated- Proof Based Hierarchical Authentication Scheme for the Internet of Things", vol. 26, no.3, Mar. 2015. 
4. V.G.T. NishanthaVidanagama and et al., "Service Environment for Smart Wireless Devices: An M2M gateway selection scheme", vol. 3, 2015.

5. K. Sutox, H. Nishiyamax and et al., "An EnergyEfficient and delay- aware wireless computing system for Industrial WSNs", pp.:2169-3536, 2015.

6. Xiaoxuan Hu, Jin Qil and et al., "A Hybrid Security and Compressive Sensing Based Sensor Data Gathering Scheme", vol.3, 2015.

7. C. Harold Liu \& S. Jayawardena and et al., "The Emerging IoT Market place from an Industrial Perspective: A Survey". IEEE, Jan. 2015.

8. A. Zanella, and etc., "Internet of Things for Smart Cities", IEEE Internet of Things, Feb. 2014.

9. S. Rajguru and et al., "Analysis of IoT in a Smart Environment", vol. 4, Issue 4, pp.:40-43, April. 2015.

10. Amrita VishwaVidyaPeetham, Amrita center for wireless networks and applications, 2013.

11. Menon1, et al., "Implementation of internet of things in bus transport system of Singapore", Asian Journal of Engineering Research, 2013.

12. Shao-Lei Zhai et al, "Research of Communication Technology on IOT for High-Voltage Transmission Line", International Journal of Smart Grid and Clean Energy, 2012.

13. S. Pandikumar and et al., "IoT Based Architecture of Web and Smart Home Interface Using GSM", International Journal of Innovative Research in Science, Engineering and Technology, vol. 3, March 2014.

14. O. Anusha, CH. Rajendra Prasad, "Experimental Investigation on Road Safety System at Crossings", IJEAT, vol.8, Issue-2S2, pp.214-218, January 2019.

15. K. Barath Reddy and Ch. Rajendraprasad, "The embedded Web server based Electrical Ethernet Monitoring system using ARM," Int. Jr. of Adv. Research in Comp. \& Comm. Engg. Vol. 2, Issue 5, pp. 2292-2295, 2013,

16. N. Deepak, Ch. Rajendra Prasad "Patient Health Monitoring using IoT”, IJITEE, vol.8, Issue-2S2, Dec.2018.

17. P.RamchanderRao, S. Sanjay Kumar and Ch. Rajendra Prasad "Garbage Monitoring System using Arduino", International Journal of Trend in Scientific Research and Development, Vol.1 Issue 6, 2017, pp.808-811. 